\title{
Model Quality Estimation in Systems with Time-Varying Time-delay Uncertainty
}

\author{
Soheil Salehpour and Andreas Johansson
}

\begin{abstract}
The problem of estimating bounds for timevarying parameter perturbations using measurement data is addressed. In particular, time-varying time-delay is considered. An estimate of the perturbation is produced based on a quantized approximation of the uncertainty and the sparse structure of its derivative. The Padé-approximation and orthogonal collocation method are used to approximate the delay. A first-order system with time-delay is used as an illustrative example. The gain, time-constant and time-delay are considered as uncertainties here.
\end{abstract}

Index Terms-Model quality estimation, time-varying parameter uncertainty, MILP, sparsity, perturbation, uncertainty, optimization, time-delay.

\section{INTRODUCTION}

$\mathbf{T}$ HE uncertainties associated with the nominal process model is a concern in most approaches to feedback control. The question is how to achieve a tight bound or shape of the uncertainty by using a set of measurement data. This active research area is known as model quality estimation. In the existing approaches to model quality estimation, the true system, see e.g. [1], [2], [3] and [4], is a linear time-invariant system (where uncertainty is considered in both $H_{\infty}$ and $L_{1}$ ). A time-varying linear system is a more realistic assumption, since nonlinear behavior can then also be accounted for. However, model quality estimation of time-varying perturbations appears to be difficult. Here, we consider a linear system with time-varying parameters as the model uncertainty. The prime drawback of assuming a parametric, time-varying uncertainty description is its possible shortcoming for describing unmodeled dynamics. However, if the physics of process is reasonably well-known, then the unmodeled dynamics can be limited to high frequencies, which then can often be described by a parametric model.

We will assume a state-space system with affine dependence on the uncertain time-varying parameters. This structure is frequently used in robust control and estimation [8] and [9], and can be expressed by using the Kronecker product as [5]

$$
\begin{aligned}
x(k+1)= & A x(k)+B u(k)+P(\pi(k) \otimes x(k))+ \\
& D \pi(k)+Q(\pi(k) \otimes u(k)) \\
y(k)= & C x(k)+\eta(k)
\end{aligned}
$$

where $\otimes$ denote the Kronecker product and $A \in R^{n \times n}, B \in$ $R^{n \times p}, C \in R^{l \times n}, D \in R^{n \times q}, P \in R^{n \times n q}, Q \in R^{n \times q p}$ are constant matrices. The vector $x(k) \in R^{n}$ represents the state, $y(k) \in R^{l}$ is the measured output and $u(k) \in R^{p}$ is

Andreas Johansson and Soheil Salehpour are with the Control Engineering Group, Luleå University of Technology, SE-971 87 Luleå, Sweden.

soheil@ltu.se, andreas.johansson@ltu.se the input while $\eta(k) \in R^{l}$ is some additive disturbance. The vector $\pi(k) \in R^{q}$ represents the parameter uncertainties, i.e. the deviation from the nominal parameter values. Note that they are allowed to enter both linearly and and bilinearly with the state $x$ as well as with the input $u$.

In [14], we present two methods to estimate bounds of parameter uncertainty in state-space systems. The methods are based on estimates of the perturbations from measurement data. Since the number of perturbations is usually larger than the number of measurement signals $(q>l)$, this requires additional assumptions on the uncertainty. In [14], we firstly assume that the perturbation and its derivative is minimal. Then, we suppose the perturbation is minimal and its derivative is maximally sparse. This later approach is proved to be particularly fruitful.

In discrete-time systems, a time-delay is described using extra state variables [15], which raises difficulties when considering a time-varying time-delay. Instead, we will use a sampled continuous-time approximation. Then, we use the method based on optimization of the sparseness of the derivative of the quantized perturbation to estimate the variation of the time-delay.

In Section II, an approximation of (1) is given and a bound for the disturbance is defined. A MILP (Mixed Integer Linear Programming) algorithm to maximize the sparsity of a matrix is described and a method based on this algorithm estimates the perturbations. In section III, we investigate the use of Euler discretization of a realization of the delay based on Padé- and OC- approximations. A first-order process model with time-delay which is widely used in industry is considered as an example in Section IV and simulation results are given in Section V. Section VI gives some concluding remarks and directions for future work.

\section{PRELIMINARIES}

The vector of dimension $n$ where each element is 1 is denoted as $1_{n}=[1,1, \ldots, 1]^{T}$ and $I_{n}$ is the identity matrix of dimension $n$.

\section{A. The Biaffine Input/Output System}

In this section, we give an approximation of (1) whose output is affine in the input $u$ and perturbation $\pi$. It is derived in [10] as a 1'st order Taylor approximation of $y$ with respect to $\pi$ and may be expressed as

$$
\begin{aligned}
\zeta(k+1)= & A \zeta(k)+B u(k) \\
\xi(k+1)= & A \xi(k)+P(\pi(k) \otimes \zeta(k))+ \\
& D \pi(k)+Q(\pi(k) \otimes u(k)) \\
\hat{y}(k)= & C(\zeta(k)+\xi(k))
\end{aligned}
$$


with initial state $\zeta(0)=x(0)$ and $\xi(0)=0$. By defining the disturbance $\nu=r+\eta$, where $r$ denotes the linearization error, the output of (1) may be expressed as

$$
y(k)=\hat{y}(k)+\nu(k)
$$

Furthermore, by collecting the signals into vectors as

$$
\begin{aligned}
\Pi & =\left[\pi(0)^{T} \pi(1)^{T} \cdots \pi(N-1)^{T}\right]^{T} \\
Y & =\left[y(0)^{T} y(1)^{T} \cdots y(N)^{T}\right]^{T} \\
V & =\left[\nu(0)^{T} \nu(1)^{T} \cdots \nu(N)^{T}\right]^{T}
\end{aligned}
$$

(2) may also be formulated [10] as

$$
Y=\Upsilon+\Xi \Pi+V
$$

where

$$
\begin{aligned}
\Xi & =\left[\begin{array}{cccc}
0 & 0 & \cdots & 0 \\
C \phi(0) & 0 & \cdots & 0 \\
C A \phi(0) & C \phi(1) & \cdots & 0 \\
\vdots & \vdots & \ddots & \vdots \\
C A^{N-2} \phi(0) & C A^{N-3} \phi(1) & \ddots & 0 \\
C A^{N-1} \phi(0) & C A^{N-2} \phi(1) & \cdots & C \phi(N-1)
\end{array}\right] \\
\Upsilon & =\left[\begin{array}{c}
C \zeta(0) \\
C \zeta(1) \\
\vdots \\
C \zeta(N)
\end{array}\right]
\end{aligned}
$$

and $\phi(i)=P\left(I_{q} \otimes \zeta(i)\right)+Q\left(I_{q} \otimes u(i)\right)+D$ for $i=$ $0, \ldots, N-1$.

\section{B. Disturbance Bound}

To bound the disturbance $\nu(k)$ we will use the window norm, which for continuous time signals is defined as [11]

$$
\|\nu\|_{\omega}=\sup _{t \geq 0} \int_{0}^{t} \omega(t-\tau)|\nu(\tau)| d \tau
$$

where $\omega(t)$ is nonnegative and bounded by an exponentially decreasing function. In [10] a discrete time window norm is defined as follows

Definition 1: A window sequence is a sequence $\omega: Z^{+} \rightarrow$ $R^{+}$, which is not identically zero and satisfies $\omega(k) \leq c e^{-a k}$ for all $k \geq 0$ and some positive $c$ and $a$.

Given a window sequence $\omega(k)$, the window norm for a discrete time signal $\nu(k)$ may then be defined as

$$
\|\nu\|_{\omega}=\sup _{k \geq 0} \sum_{i=0}^{k} \omega(k-i)|\nu(i)|
$$

It is remarked that (5) satisfies all properties of a norm, but the proof is omitted. It is straightforward to see that the window norm is equal to the $l_{\infty}$-norm if one chooses $\omega$ as the unit pulse function. The drawback of $l_{\infty}$-norm is that it only considers the peak value of the signal without any averaging and may therefore be conservative. This problem is handled in the window norm by choosing a window function that averages over a suitable time interval, i.e. a pulse function with non-unit duration or a decaying exponential function.
The window norm actually approaches the $l_{1}$-norm by letting $\omega$ approach a unit step function. An assumption on the disturbance $\nu=\left[\nu_{1}, \ldots, \nu_{l}\right]$ may now be expressed as

$$
\left\|\nu_{j}\right\|_{\omega} \leq \epsilon_{j}, j=1, \ldots, l
$$

We assume that each $\omega(k)$ is monotone decreasing for $k>0$. Then, as showed in [10] for scalar $\nu$, the condition (6) can be expressed as

$$
W|V| \leq 1_{N+1} \otimes \epsilon
$$

where

$$
W=\left[\begin{array}{cccc}
\omega(0) & 0 & \cdots & 0 \\
\omega(1) & \omega(0) & \cdots & 0 \\
\vdots & \vdots & \ddots & \vdots \\
\omega(N) & \omega(N-1) & \cdots & \omega(0)
\end{array}\right]
$$

and $\epsilon=\left[\epsilon_{1}, \epsilon_{2}, \ldots, \epsilon_{l}\right]^{T}$.

\section{Sparse Matrix}

In numerical analysis, a sparse matrix is a matrix populated primarily with zeros. The concept of sparsity is useful in complex systems and many application areas such as network theory. Huge sparse matrices often appear in science or engineering when solving partial differential equations.

One common approach to seeking a sparse description is based on $l_{1}$-norm regularization [6] which produces an approximation with a sparse structure.

In this article, we present an exact solution of maximization of sparsity by using MILP (Mixed Integer Linear Programming) to minimize the number of non-zero elements in a matrix or vector.

We consider the logical variable $\delta_{i j} \in\{0,1\}$ and the matrix $S=\left(s_{i j}\right)_{i, j=1 \ldots N}$ to be related as

$$
\begin{aligned}
& \delta_{i j}=1 \leftrightarrow s_{i j} \neq 0 \\
& \delta_{i j}=0 \leftrightarrow s_{i j}=0
\end{aligned}
$$

and we aim to minimize $\sum_{i, j=1}^{N} \delta_{i j}$ for $i, j=1, \ldots, N$. First, we assume $\delta_{i j}=\delta_{i j}^{1}+\delta_{i j}^{2}$ and (8) is re-written as

$$
\begin{aligned}
& \left(\delta_{i j}^{1}, \delta_{i j}^{2}\right)=(1,0) \leftrightarrow s_{i j}>0 \\
& \left(\delta_{i j}^{1}, \delta_{i j}^{2}\right)=(0,0) \leftrightarrow s_{i j}=0 \\
& \left(\delta_{i j}^{1}, \delta_{i j}^{2}\right)=(0,1) \leftrightarrow s_{i j}<0
\end{aligned}
$$

The above link between logical and real variables can also be expressed by using MILP inequalities

$$
\begin{gathered}
m \delta_{i j}^{1}-M \delta_{i j}^{2} \leq s_{i j} \leq M \delta_{i j}^{1}-m \delta_{i j}^{2} \\
\delta_{i j}^{1}+\delta_{i j}^{2} \leq 1
\end{gathered}
$$

where $m=\min _{i, j}\left(\left|s_{i j}\right|\right)$ and $M=\max _{i, j}\left(\left|s_{i j}\right|\right)$. Then, a MILP for maximizing sparsity of $S$ is formulated as

$$
\begin{array}{cc}
\operatorname{minimize} & \sum_{i, j=1}^{N}\left(\delta_{i j}^{1}+\delta_{i j}^{2}\right) \\
\text { subject to: } & (9)
\end{array}
$$

Here, we also present an approximation method which is more practical in a numerical sense. This method instead 
minimizes the number of elements of a matrix which are larger than a certain threshold. If we consider $\mu$ as threshold, the logical variables are changed into

$$
\begin{aligned}
& \delta_{i j}=1 \leftrightarrow\left|s_{i j}\right|>\mu \\
& \delta_{i j}=0 \leftrightarrow\left|s_{i j}\right| \leq \mu
\end{aligned}
$$

Then, the inequalities (9) are substituted in (10) by

$$
\begin{aligned}
2 \mu \delta_{i j}^{1}-M \delta_{i j}^{2}-\mu & \leq s_{i j} \leq M \delta_{i j}^{1}-2 \mu \delta_{i j}^{2}+\mu \\
\delta_{i j}^{1}+\delta_{i j}^{2} & \leq 1
\end{aligned}
$$

where $M=\max _{i, j}\left(\left|s_{i j}\right|\right)+\mu$ and $\mu$ is a small number.

\section{Method Based on MILP and the Derivative of Uncer- tainty}

Some assumptions on the perturbations are required in order to estimate them. We assume that the perturbations are small and that the disturbance $\nu$ is also small. The size of the perturbation $\pi$ is measured with $\sup _{k}\|F \pi(k)\|_{\infty}$, where $\|.\|_{\infty}$ is the $l_{\infty}$ vector norm and $F$ is a diagonal weight matrix. Scaling of the vectorized perturbation $\Pi$ is accomplished by

$$
(I \otimes F) \Pi=\left[(F \pi(0))^{T}(F \pi(1))^{T} \cdots(F \pi(N-1))^{T}\right]^{T}
$$

Our next assumption is that the perturbations are due to deterministic physical phenomena and thus have a nonrandom nature. In particular, we assume that the perturbation can be modeled as $\pi=L \sigma$, where $\sigma$ is a series of pulses, representing deterministic real-world events and $L$ is some linear system, in our case simply an integrator. This means that the perturbations can be approximated by quantization. Quantization as a means of approximating signals is widely used in digital control and other areas. The differentiation of a quantized signal produces a lot of zero samples and thus, it has a sparse structure. The time-derivative of $\pi$ is approximated by $(\Delta \pi)(k)=\pi(k)-\pi(k-1)$. Now, With the definitions

$$
\begin{aligned}
\sigma_{i} & =\left[\delta_{1 i}^{1}, \delta_{1 i}^{2}, \cdots, \delta_{q i}^{1}, \delta_{q i}^{2}\right] \text { for } i=0, \cdots, N-1, \\
\Sigma_{N} & =\left[\sigma_{0}, \sigma_{1}, \cdots, \sigma_{N-1}\right]^{T}, U=I_{N q} \otimes[M,-2 \mu], \\
L & =I_{N q} \otimes[2 \mu,-M] \text { and } \Lambda=I_{N q} \otimes[1,1]
\end{aligned}
$$

the sparsity constraint (11) applied to the derivative $\Delta \Pi$ may be expressed as

$$
\begin{aligned}
L \Sigma_{N}-1_{N q} \mu & \leq \Delta \Pi \leq U \Sigma_{N}+1_{N q} \mu \\
\Lambda \Sigma_{N} & \leq 1_{N q}
\end{aligned}
$$

where $\Delta \Pi=\left[\Delta \pi(0)^{T} \Delta \pi(1)^{T} \cdots \Delta \pi(N-1)^{T}\right]^{T}$. Also, let $Y_{m}=\left[y_{m}(0)^{T} y_{m}(1)^{T} \cdots y_{m}(N)^{T}\right]^{T}$, where $y_{m}(k)$ are the measured outputs of (1). We now use the constraint (12), and MILP-optimization to formulate

$$
\begin{array}{cc}
\underset{\Sigma_{N}, \Pi}{\operatorname{minimize}} & 1_{2 N q} \Sigma_{N}+\|(I \otimes F) \Pi\|_{\infty}+H \epsilon \\
\text { subject to: } & (12) \\
& W\left|Y_{m}-\Upsilon-\Xi \Pi\right| \leq \epsilon
\end{array}
$$

where $H$ is a weight for the disturbance.

\section{ApPROXIMATION OF A TIME-DELAY}

The Padé- and orthogonal collocation-(OC-) approximation are commonly used to approximate a time-delay ( [12] and [13]). If an approximation of a time-delay in statespace form is given, then we can realize another time-delay by time-scaling of the step-response using the following proposition.

Proposition: Consider a linear system on state-space form

$$
\begin{aligned}
& \dot{x}(t)=A x(t)+B u(t) \\
& y(t)=C x(t)+D u(t)
\end{aligned}
$$

with step-response $G(t)$. Then a linear system with stepresponse $G(t / \tau)$ is given by

$$
\begin{aligned}
\dot{x}(t) & =\frac{A}{\tau} x(t)+\frac{B}{\tau} u(t) \\
y_{\tau}(t) & =C x(t)+D u(t)
\end{aligned}
$$

\section{Proof: See appendix}

This means e.g. that if (14) is an approximation of a 1 second time-delay. Then, an approximation of the arbitrary time-delay $\tau$ may be realized as (15). An Euler approximation of (15) with sampling interval $h$ is

$$
x(k+1)=\left(I+\frac{A}{\tau} h\right) x(k)+\frac{B}{\tau} h u(k)
$$

Now, we explore the effect of the Euler discretizing on the frequency response and stability of the Padé- and OCapproximations. The poles of the discrete-time model (16) are the eigenvalues of $\left(I+\frac{A}{\tau} h\right)$ which depend on the choice of $h, \tau$ and the order of the Padé- and OC- methods. A nominal delay of the model is assumed to be given and we often choose a large order to get better approximation of the delay with respect to phase difference. So, we try to find a value for the sampling-time which guarantees stability of (16).

The Bode-diagram and pole-zero map of the Padé- and OC- approximations for different sampling intervals with order 7 and $\tau=10$ are depicted in Fig. 1 and Fig. 2. Fig. 1 shows a good correspondence between the continuous models and the discretized Padé- and OC- approximations for frequencies under 0.2. As showed in Fig. 2, the OCand Padé- methods are unstable for sampling intervals over 0.3 and 0.7 , respectively. The sampling interval in our simulations are chosen to be $h=0.2$ seconds.

\section{Applichtion to A First-ORder Process MOdel WITH TIME-DELAY}

In process control, it is common to approximate the process with a first-order system with time-delay as

$$
G(s)=\frac{K}{1+T s} e^{-\tau s}
$$

where $K, T$ and $\tau$ are respectively the gain, time-constant and time-delay of the process. We will apply the methods to such a process with measurement $y_{c}$, i.e.

$$
\dot{y}_{c}=-\frac{1}{T} y_{c}+\frac{K}{T} u(t-\tau)
$$


and the state-space form of $u(t-\tau)$ is approximated as

$$
\begin{aligned}
\dot{x}_{1}(t) & =\frac{A_{0}}{\tau} x_{1}(t)+\frac{B_{0}}{\tau} u(t) \\
u(t-\tau) & =C_{0} x_{1}(t)+D_{0} u(t)
\end{aligned}
$$

where $A_{0}, B_{0}, C_{0}$ and $D_{0}$ represent an approximate realization of a 1 second time-delay. An Euler approximation gives the following discrete-time system

$$
\begin{aligned}
x_{1}(k+1) & =\left(I+\frac{A_{0}}{\tau} h\right) x_{1}(k)+\frac{B_{0}}{\tau} h u(k) \\
x_{2}(k+1) & =\left(1-\frac{h}{T}\right) x_{2}(t)+\frac{K h}{T}\left(C_{0} x_{1}(k)+D_{0} u(k)\right) \\
y(k) & =x_{2}(k)
\end{aligned}
$$

where $u(k)=u_{c}(k h)$ and $y(k)=y_{c}(k h)$.

We introduce two forms for parameterization of the uncertainties $(T, K$ and $\tau$ ). The first form is

$$
\begin{aligned}
T(k) & =T_{0}\left(1+\pi_{1}(k)\right), \quad K(k)=K_{0}\left(1+\pi_{2}(k)\right) \\
\tau(k) & =\tau_{0}\left(1+\pi_{3}(k)\right)
\end{aligned}
$$

A 1st order Taylor approximation of the right-hand side of (17) with respect to $\pi_{1}, \pi_{2}$ and $\pi_{3}$ is

$$
\begin{aligned}
x_{1}(k+1) \approx & \left(I+\frac{A_{0} h}{\tau_{0}}-\frac{A_{0} h}{\tau_{0}} \pi_{3}(k)\right) x_{1}(k) \\
& +\left(\frac{B_{0} h}{\tau_{0}}-\frac{B_{0} h}{\tau_{0}} \pi_{3}(k)\right) u(k) \\
x_{2}(k+1) \approx & \left(1-\frac{h}{T_{0}}+\frac{h}{T_{0}} \pi_{1}\right) x_{2}(t) \\
& +\frac{K_{0} h}{T_{0}}\left(1-\pi_{1}+\pi_{2}\right)\left(C_{0} x_{1}(k)+D_{0} u(k)\right)
\end{aligned}
$$

Thus, with the definitions

$$
\begin{aligned}
& A=\left[\begin{array}{cc}
I+\frac{A_{0} h}{\tau_{\varphi}} & 0 \\
\frac{K_{0} C_{0} h}{T_{0}} & 1-\frac{h}{T_{0}}
\end{array}\right], B=\left[\begin{array}{c}
\frac{B_{0} h}{\tau_{0}} \\
\frac{K_{0} D_{0} h}{T_{0}}
\end{array}\right] \\
& P=\left[\begin{array}{cccccc}
0 & 0 & 0 & 0 & -\frac{A_{0} h}{\tau_{0}} & 0 \\
-\frac{K_{0} C_{0} h}{T_{0}} & \frac{h}{\tau_{0}} & \frac{K_{0} C_{0} h}{T_{0}} & 0 & 0 & 0
\end{array}\right] \\
& D=\left[\begin{array}{lll}
0 & 0 & 0 \\
0 & 0 & 0
\end{array}\right], Q=\left[\begin{array}{ccc}
0 & 0 & -\frac{B_{0} h}{\tau_{0}} \\
-\frac{K_{0} D_{0} h}{T_{0}} & \frac{K_{0} D_{0} h}{T_{0}} & 0
\end{array}\right]
\end{aligned}
$$

the process may be stated as (1).

To avoid the Taylor approximation, we also consider the following parameterization of the uncertainty

$$
\begin{aligned}
& \frac{1}{T(k)}=\frac{1}{T_{0}}+\pi_{1}(k), \frac{K(k)}{T(k)}=\frac{K_{0}}{T_{0}}+\pi_{2}(k) \\
& \frac{1}{\tau(k)}=\frac{1}{\tau_{0}}+\pi_{3}(k)
\end{aligned}
$$

Then (17) is re-written as

$$
\begin{aligned}
x_{1}(k+1)= & \left(I+\frac{A_{0} h}{\tau_{0}}+A_{0} h \pi_{3}(k)\right) x_{1}(k) \\
& +\left(\frac{B_{0} h}{\tau_{0}}+B_{0} h \pi_{3}(k)\right) u(k) \\
x_{2}(k+1)= & \left(1-\frac{h}{T_{0}}-h \pi_{1}\right) x_{2}(t) \\
& +h\left(\frac{K_{0}}{T_{0}}+\pi_{2}\right)\left(C_{0} x_{1}(k)+D_{0} u(k)\right)
\end{aligned}
$$

With the definitions

$$
\begin{aligned}
A & =\left[\begin{array}{cc}
I+\frac{A_{0} h}{\tau_{h}} & 0 \\
\frac{K_{0} C_{0} h}{T_{0}} & 1-\frac{h}{T_{0}}
\end{array}\right], B=\left[\begin{array}{c}
\frac{B_{0} h}{T_{0}} \\
\frac{K_{0} D_{0} h}{T_{0}}
\end{array}\right] \\
P & =\left[\begin{array}{cccccc}
0 & 0 & 0 & 0 & A_{0} h & 0 \\
0 & -h & C_{0} h & 0 & 0 & 0
\end{array}\right] \\
D & =\left[\begin{array}{lll}
0 & 0 & 0 \\
0 & 0 & 0
\end{array}\right], Q=\left[\begin{array}{ccc}
0 & 0 & B_{0} h \\
0 & D_{0} h & 0
\end{array}\right]
\end{aligned}
$$

the process may be expressed as (1).

\section{Simulation Results}

The MILP-optimization algorithm (13) is applied to the process (17) with uncertainties parameterized as (18) and (19). Padé- and OC- approximations are utilized to approximate the delay, i.e. provide $A_{0}, B_{0}, C_{0}$ and $D_{0}$ in (17). The time-varying parameters are depicted in Fig. 4 as thick, solid lines and their nominal parameter values $T_{0}, K_{0}$ and $\tau_{0}$ are chosen 10, 5 and 10, respectively. The MILP-optimization method is implemented in the YALMIP software [7] and applied to the process. Weighting factors are chosen as $F=I_{3}$ and $F=\operatorname{diag}\left(T_{0}, \frac{T_{0}}{K_{0}}, \tau_{0}\right)$ for parameterization (18) and (19), respectively. We take $H=1 /\left\|y_{m}-C \zeta\right\|_{\omega}$ for both forms.

First, we test the process with the step input in Fig. 3(a). The order of the Padé- and OC- approximations are chosen to be 7 . The results are depicted in Fig. 4, which shows good tracking of the gain $(K)$ and time-delay $(\tau)$, but the algorithms do not detect the variation of the time-constant (T).

In order to improve the result, we consider a combination of sinusoidal inputs with frequencies under 0.2 (Fig. 3(a)). The simulation results are showed in Fig. 5. All the methods except when we use (19) in combination with the OCapproximation, detect the change of $T$, and the best tracking is achieved by using (19) in combination with the Padéapproximation. Wherever we use (19), a variation of $T$ is obtained when $K$ changes. The reason is that $K / T$ is a parameter in these cases, i.e. the variation of $K$ is compensated by a change of $T$.

To improve the result further, we increase the order of the Padé- and OC- approximations. The results for order 12 are showed in Fig. 6, and it can be seen that all methods detect the change of $T$, though we have more oscillation in $T, K$ and $\tau$. For input signal frequencies over 0.2 or higher order than 12 of Padé- and OC- method we get oscillating behavior of the time-delay approximation, resulting in deteriorated results.

\section{CONCLUSIONS AND FUTURE WORK}

An optimization approach is presented to estimate the time-varying parameter uncertainty in state-space system. Particularly, the case of time-varying time-delay uncertainty is addressed. A first-order system with time-delay is used as illustrative example. Two parameterizations of the perturbations and two approximation methods (Padé- and OCapproximations) are considered.

The promising results of the MILP method shows that exploiting the non-randomness of the perturbations is a 


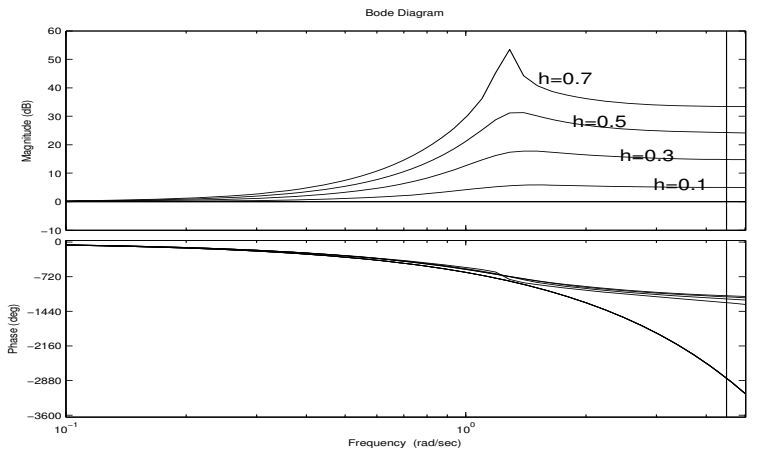

(a)

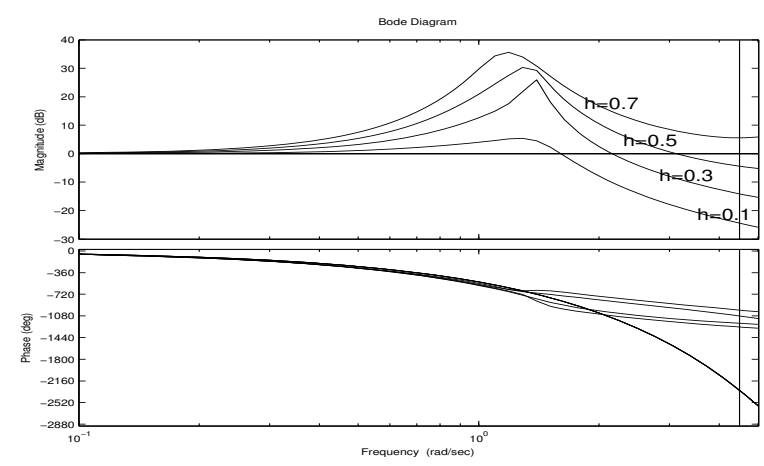

(b)

Fig. 1. Bode-diagram of (a) Padé- and (b) OC- approximations with order $7, \tau=10$ and $h=0.1,0.3,0.5,0.7$.

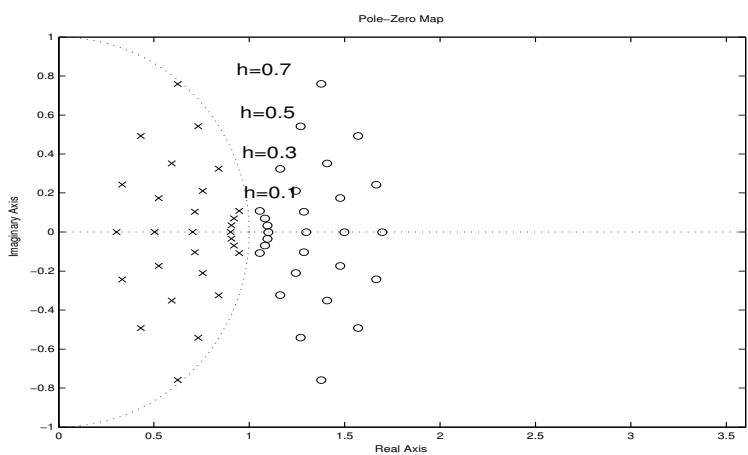

(a)

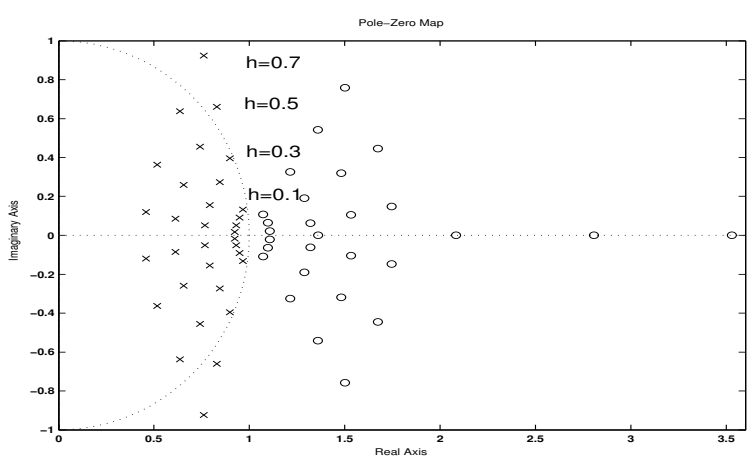

(b)

Fig. 2. Pole-zero map of (a) Padé- and (b) OC- approximations with order $7, \tau=10$ and $h=0.1,0.3,0.5,0.7$.

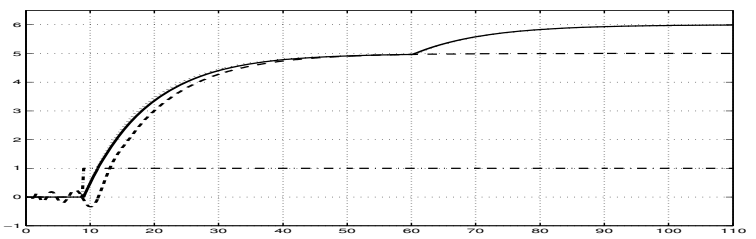

(a)

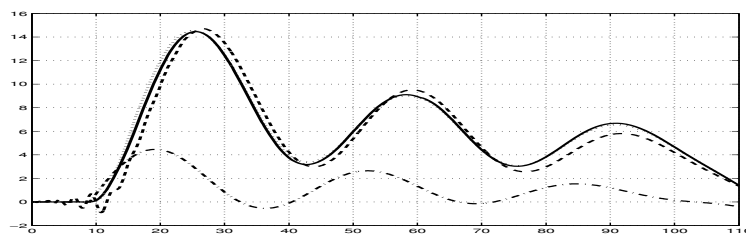

(b)

Fig. 3. (y) Output of (1) (solid line), (u) input (-.), nominal output of (2)(- -), output of (2) (...). (a) Step input (b) A combination of sinusoidal input.

viable approach to the estimating time-varying parameter uncertainty.

For future research, a more systematic way choose appropriate sampling-time and order of Padé- and OCapproximations will be investigated. To improve the estimation, other properties of noise and perturbations may also be exploited, e.g. that the noise is expected to be random and uncorrelated with the perturbations.

\section{ACKNOWLEDGMENTS}

The authors wish to thank the Hjalmar Lundbohm Research Center (HLRC) funded by LKAB for financing this research.

\section{APPENDIX}

The impulse-response of (14) is

$$
g_{1}(t)=C e^{A t} B+D \gamma(t)
$$

with corresponding step response

$$
G_{1}(t)=\int_{0}^{t} g_{1}(p) d p=C A^{-1} e^{A t} B+D \theta(t)
$$

where $\gamma$ and $\theta$ represent the Dirac impulse and the Heaviside step, respectively. The time-scaled $G_{1}(t)$ is

$$
G_{\tau}=G_{1}\left(\frac{t}{\tau}\right)=C A^{-1} e^{A t / \tau} B+D \theta(t)
$$

and the the corresponding impulse response is given by the time derivative

$$
g_{\tau}(t)=C e^{\frac{A}{\tau} t} \frac{B}{\tau}+D \gamma(t)
$$

which has the realization (15).

\section{REFERENCES}

[1] L. Giarre, M. Milanese and M. Taragna, $H_{\infty}$ identification and model quality estimation. IEEE Transaction on Automatic Control, 42(2): 188199, 1997.

[2] M. Milanese and M. Taragna. $H_{\infty}$ set membership identification: A survey. Automatica, 41: 2019-2032, 2005.

[3] W. Reinelt, A. Garulli and L. Ljung. Comparing different approaches to model error modeling in robust identification. Automatica, 38:787-803, 2002. 

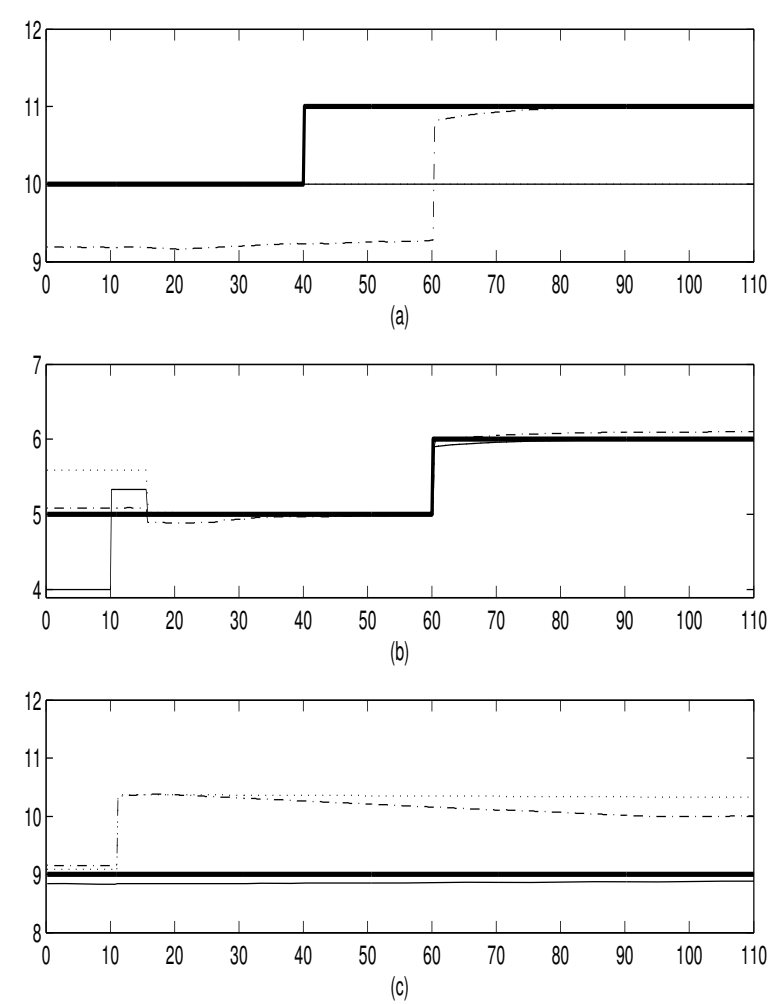

Fig. 4. Step input. Estimates of perturbations $\pi_{1}, \pi_{2}$ and $\pi_{3}$ in (a), (b) and (c), respectively. True perturbations (thick solid line), (18) and OCapproximation (...), (18) and Padé-approximation (solid line), (19) and OCapproximation (- - -), (19) and Padé-approximation (-.).
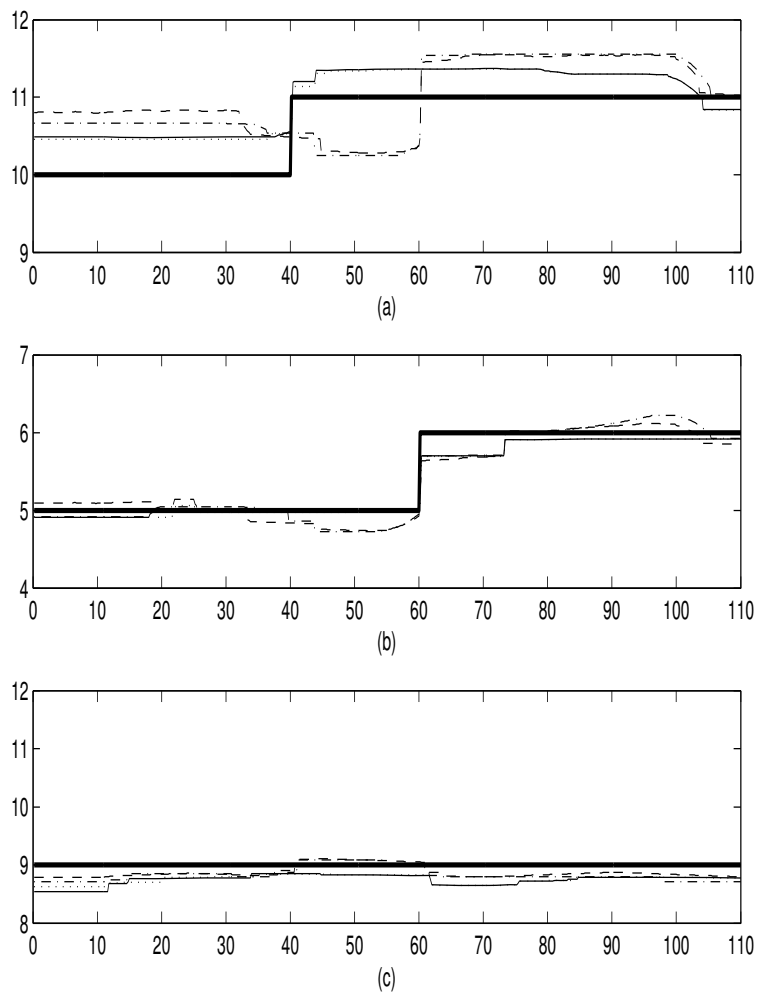

Fig. 5. Sinusoidal inputs. Estimates of perturbations $\pi_{1}, \pi_{2}$ and $\pi_{3}$ in (a), (b) and (c), respectively. True perturbations (thick solid line), (18) and OC-approximation (...), (18) and Padé-approximation (solid line), (19) and OC- approximation (- - -), (19) and Padé-approximation (-.).
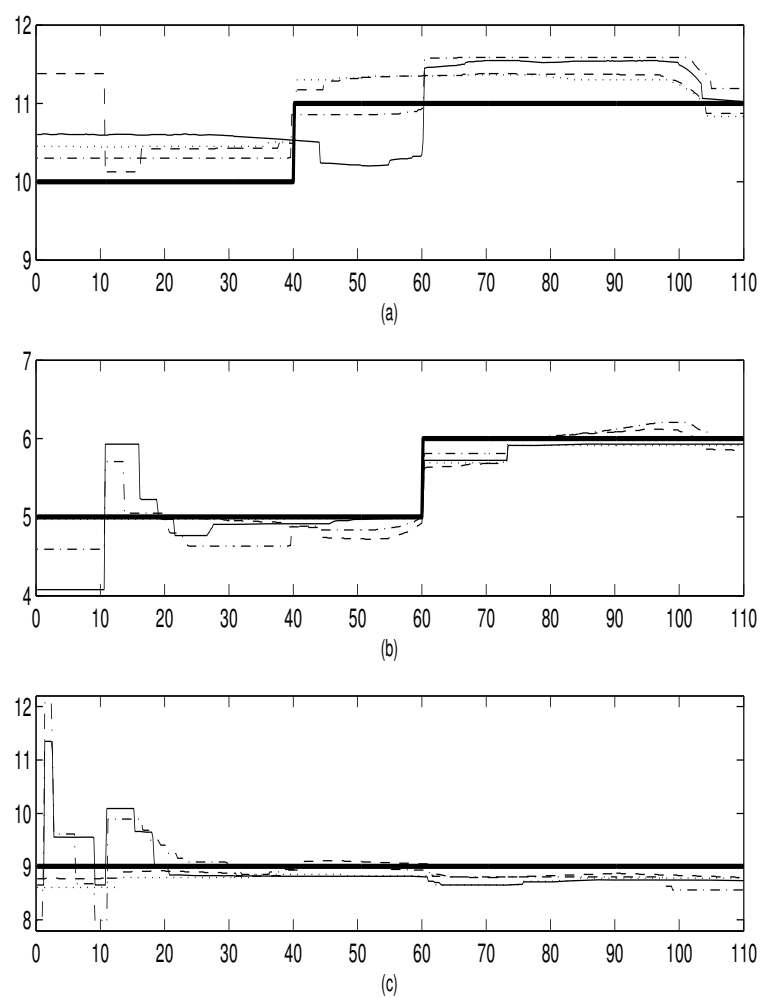

Fig. 6. Sinusoidal inputs, $12^{\text {th }}$-order time-delay approximation. Estimates of perturbations $\pi_{1}, \pi_{2}$ and $\pi_{3}$ in (a), (b) and (c), respectively. True perturbations (thick solid line), (18) and OC-approximation (...), (18) and Padé-approximation (solid line), (19) and OC-approximation (- - -), (19) and Padé-approximation (-.).

[4] V.F. Sokolov. Control oriented model validation and errors quantification in the $l_{1}$ setup. IEEE Transactions on Automatic Control, 50(10): 1501-1508, 2005

[5] A. Johansson, M. Bask and T. Norlander. Dynamic threshold generators for robust fault detection in linear system with parameter uncertainty. Automatica 42: 1095-1106, July 2006.

[6] S. Boyd and L. Vandenberghe. Convex Optimization.Cambridge University, 2007 Stephen Boyd (with Lieven Vandenberghe, 2004)

[7] J. Löfberg. YALMIP : A Toolbox for Modeling and Optimization in MATLAB. In Proceedings of the CACSD Conference, Taipei, Taiwan, 2004.

[8] Carlos E. de Souza, K. A. Barbosa and A. Trofino. Robust filtering for linear systems with convex-bounded uncertain time-varying parameters. IEEE Transactions on Automatic Control, 52(6): 1132-1138, 2007.

[9] D. Xie and G. Xu. Computation of performance robustness bounds for control systems with parameter uncertainties: An LMI approach. IEEE Transactions on Automatic Control Appl., 152(6): 675-682, 2005.

[10] A. Johansson. Validation of state-space models with time-varying parameter uncertainty. In Proceedings of the $46^{\text {th }}$ IEEE Conference on Decision and Control, New Orleans, USA, 2007.

[11] A. Johansson. Shift-invariant signal norms for fault detection and control. System and Control Letters, 57(2008), 105-111.

[12] G. H. Golub and C. F. Van Loan, Matrix Computations, Johns Hopkins University Press, Baltimore, 1989, pp. 557-558.

[13] L. Ding, T. Gustafsson and A. Johansson. Model parameter estimation of simplified linear models for a continuous paper pulp digester, Journal of Process Control, Volume 17, Issue 2, February 2007, Pages 115-127.

[14] S. Salehpour and A. Johansson. Two Algorithms for Model Quality Estimation in State-Space Systems with Time-Varying Parameter Uncertainty. To be presented at the American Control Conference in Seattle, USA, 2008.

[15] K. J. Åström and B. Wittenmark. Computer-Controlled systems, Prentice-Hall, Englewood Cliffs, N.J. 07632, 1990. 